\title{
Measurement of HOMs in the RHIC RF Cavities
}

\author{
N. P. Abreu and E. M. Choi
}

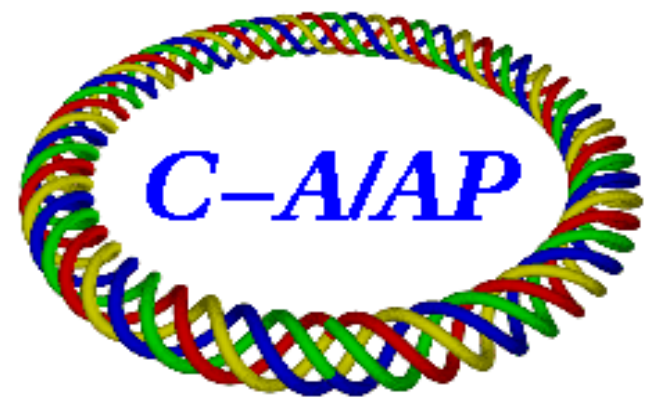

\section{Collider-Accelerator Department Brookhaven National Laboratory Upton, NY 11973}

Notice: This document has been authorized by employees of Brookhaven Science Associates, LLC under Contract No. DE-AC02-98CH10886 with the U.S. Department of Energy. The United States Government retains a nonexclusive, paid-up, irrevocable, world-wide license to publish or reproduce the published form of this document, or allow others to do so, for United States Government purposes. 
$\mathrm{C}-\mathrm{AD} / \mathrm{AP} / 320$

\title{
Measurement of HOMs in the RHIC RF cavities
}

\author{
N. P. Abreu and E. M. Choi
}

January 7, 2009

\begin{abstract}
We present results of Higher Order Modes (HOMs) measurements in the RHIC accelerating (28 $\mathrm{MHz}$ system) and storage (197 MHz system) cavities. The power of the excited HOMs deposited into the HOM damper is measured and compared with an analytical calculation of the HOMs power. The quality factors (Q) are also measured and compared to previous measurements.
\end{abstract}

\section{Introduction}

The Relativistic Heavy Ion Collider (RHIC) consists of two counter rotating beams which collide at two different experiments (PHENIX at IP8 and STAR at IP6). The particles that are accelerated range from protons to gold ions and each accelerator ring has the capability to accelerate a different specie. Table 1 summarizes the main machine parameters. The RF system on both rings has to be able to capture, accelerate and store up to 120 bunches with an average total bunch intensity of $1.0 \times 10^{9}$ particles/bunch for gold ions and $2 \times 10^{11}$ for protons. In the case of heavy ions the RF system must capture the particles at injection, accelerate them through transition and transfer the bunches from the $28 \mathrm{MHz}$ system to the $197 \mathrm{MHz}$ system. The $28 \mathrm{MHz}$ accelerating cavities operate with $300 \mathrm{kV}$ before transition and $150 \mathrm{kV}$ throughout the rest of the energy ramp for ions and the $197 \mathrm{MHz}$ system operates with $4 \mathrm{MV}$ total gap voltage. In the case of protons, since the particles are injected above transition, only acceleration and storage is needed and the $28 \mathrm{MHz} \mathrm{RF}$ cavity is used with a voltage of $300 \mathrm{kV}$ while the $197 \mathrm{MHz}$ system uses a much smaller voltage of $50 \mathrm{kV}$ only to increase the synchrotron frequecy spread and improve Landau damping. The harmonic numbers of the $28 \mathrm{MHz}$ system and the

Table 1: Machine parameters.

\begin{tabular}{ccc}
\hline \hline Parameter & \multicolumn{2}{c}{ Value } \\
& gold ions & protons \\
\hline Energy & $100 \mathrm{GeV} / \mathrm{n}$ \\
Circumference & $3833.852 \mathrm{~m}$ \\
Revolution Frequency & \multicolumn{2}{c}{$78.2 \mathrm{kHz}$} \\
Beam Frequency $(120$ bunches $)$ & $9.831 \mathrm{MHz}$ \\
rms bunch length $\left(\sigma_{t}\right)$ & \multicolumn{2}{c}{$5 \mathrm{~ns}$} \\
Gamma Transition $\left(\gamma_{t r}\right)$ & 22.8 & 25 \\
$28 \mathrm{MHz}$ System Voltage & $300-150 \mathrm{kV}$ & $300 \mathrm{kV}$ \\
$197 \mathrm{MHz}$ System Voltage & $4 \mathrm{MV}$ & $50 \mathrm{kV}$ \\
\hline \hline
\end{tabular}


$197 \mathrm{MHz}$ system are 360 and 2520 for the accelerating and the storage part, respectively. Both cavities have a HOM dampers and the $197 \mathrm{MHz}$ also have a damper for the fundamental mode since it is not active during the energy ramp for ions and protons. This report summarizes the recent measurements on the HOM power and Q factors of each $\mathrm{HOM}$ for the $28 \mathrm{MHz}$ cavities and frequencies in the $197 \mathrm{MHz}$ cavities and the results are compared with previously measured and simulated values [1, 2]. The measured power value is compared to an analytical calculation.

\section{Experimental Setup}

The measurement was performed during the 2008 proton run with two different energies and intensities:

- at injection $(\gamma=25.4)$, a single bunch with $N_{b}=1.3-1.5 \times 10^{11}$ particles and

- at store $(\gamma=106.8), 109$ bunches with $N_{b}=1.2-1.6 \times 10^{11}$ particles each.

In order to measure frequencies and power of the HOMs inside the cavities we looked at the frequency spectrum in both, the $28 \mathrm{MHz}$ cavity and $197 \mathrm{MHz}$ cavity systems, using a Spectrum Analyzer (Agilent MXA N 9020A). The power was also measured independently by a power meter which is a termination of the HOM damper line.

For the $28 \mathrm{MHz}$ system we measured the power and the quality factors of all modes. The power measured for the fundamental mode was attenuated by $60 \mathrm{~dB}$ since the HOM filter has a rejection band around its frequency. The HOM power obtained at either the power-meter or the spectrum analyzer was at least down by $21 \mathrm{~dB}$ due to losses in the cable $(1 \mathrm{~dB})$ and an attenuator $(20 \mathrm{~dB})$ used to protect the equipments. For the $197 \mathrm{MHz}$ we measured only the frequencies present in the HOM damper spectrum.

\section{Measurement of HOM frequencies in the $197 \mathrm{MHz}$ System}

The measured spectrum for one of the common $197 \mathrm{MHz}$ cavities for the Yellow and Blue rings for the cases of a single bunch and also multibunch is shown in Fig. 1. We were able to identify most of the modes predicted by the URMELT simulation [1] of the $197 \mathrm{MHz}$ cavity and a collection of the measured HOM loaded quality factor is shown in Table 2. The frequency results of the $197 \mathrm{MHz}$ cavity are in a good agreement with the simulation results.

\section{Measurement of the total HOM power, frequencies and Q factors in the $28 \mathrm{MHz}$ cavity}

The measured spectrum for two of the $28 \mathrm{MHz}$ cavities for the Yellow and Blue rings are shown in Fig. 2 for the multibunch case. A closer look of the $28 \mathrm{MHz}$ line is shown in 


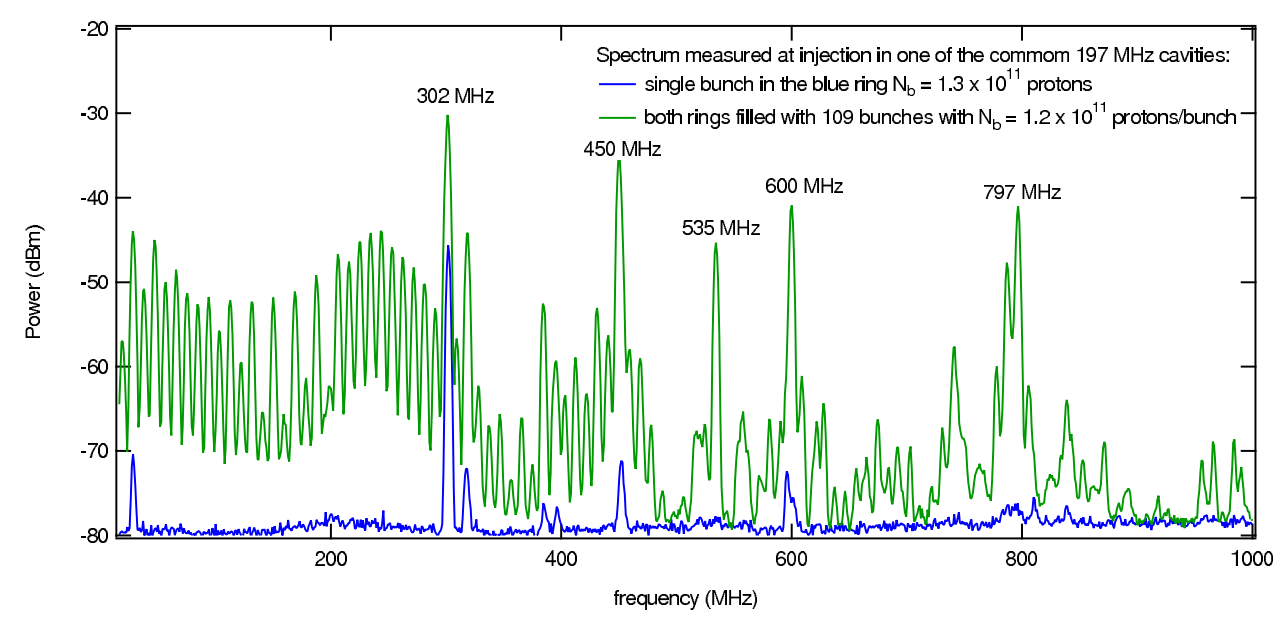

Figure 1: Measured spectrum at the HOM damper for the $197 \mathrm{MHz}$ cavity.

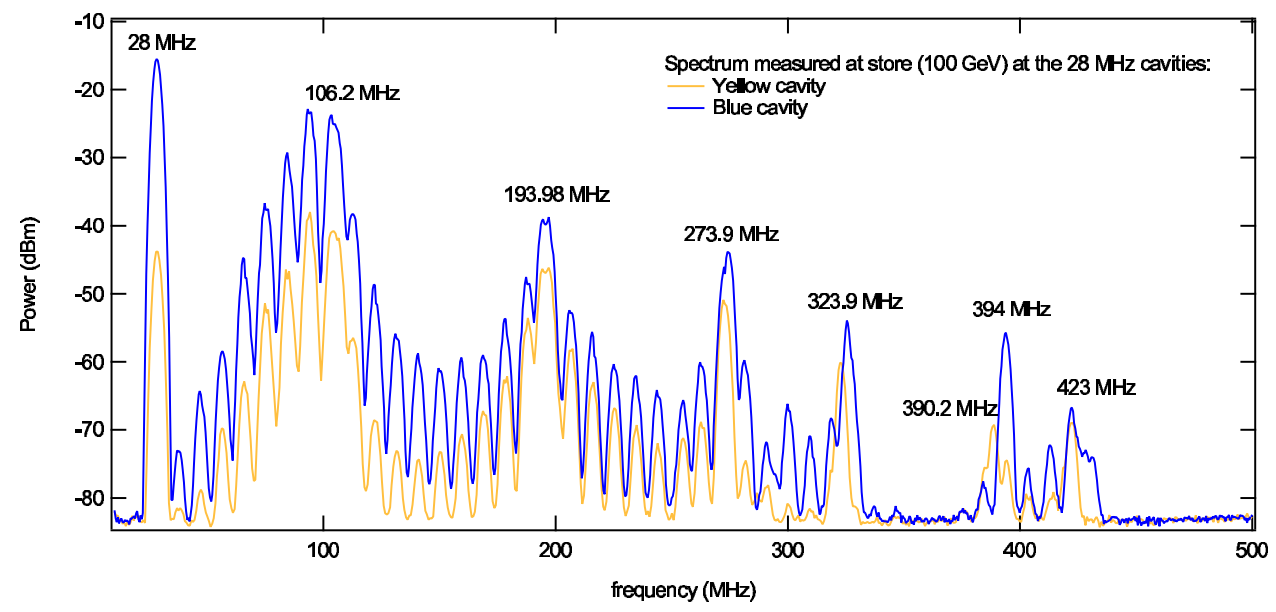

Figure 2: Measured spectrum at the HOM damper for the $28 \mathrm{MHz}$ cavity.

Fig. 3. The fundamental frequency is $28.15 \mathrm{MHz}$ and the sidebands are spaced by $78 \mathrm{kHz}$ (beam revolution frequency). Beam revolution lines are much weaker than bunch lines, which are spaced by $9.383 \mathrm{MHz}$, as shown in Fig. 3. The power was measured by means of two different equipments, first we used a power-meter, which gives the integrated HOM power, then a spectrum analyzer, which gives an individual power content of each HOM. The total power in the $28 \mathrm{MHz}$ cavities, measured using a power-meter, was $-13 \mathrm{dBm}$ for the blue cavity. Assuming that the cable loss is approximately $1 \mathrm{~dB}$ and that we were using a $20 \mathrm{~dB}$ attenuator attached just before the power-meter, the total power deposited into the HOM damper is $6.5 \mathrm{~mW}$. The summed-up total power from the individual HOM's measured for each the spectrum line shown in Fig. 2 is around $-15 \mathrm{dBm}$ for the blue cavity.Both measurements give almost a consistent output power of around $6.5 \mathrm{~mW}$. In the case of the Yellow cavity and extra attenuation of about $10 \mathrm{~dB}$ was used, that is why 
Table 2: Summary of measured HOM frequencies for the $197 \mathrm{MHz}$ and comparison with a previous measurement (196.1 MHz system) [1]

\begin{tabular}{ccc}
\hline \hline $197 \mathrm{MHz}$ & \multicolumn{2}{c}{$196.1 \mathrm{MHz}$} \\
$\mathrm{f}(\mathrm{MHz})$ & $\mathrm{f}(\mathrm{MHz})$ & $\mathrm{Q}_{L}$ \\
\hline 302 & 308.4 & 4400 \\
452 & 445 & 1200 \\
535 & 543 & 690 \\
596 & 604.2 & 1500 \\
797 & 844 & 1000 \\
\hline \hline
\end{tabular}

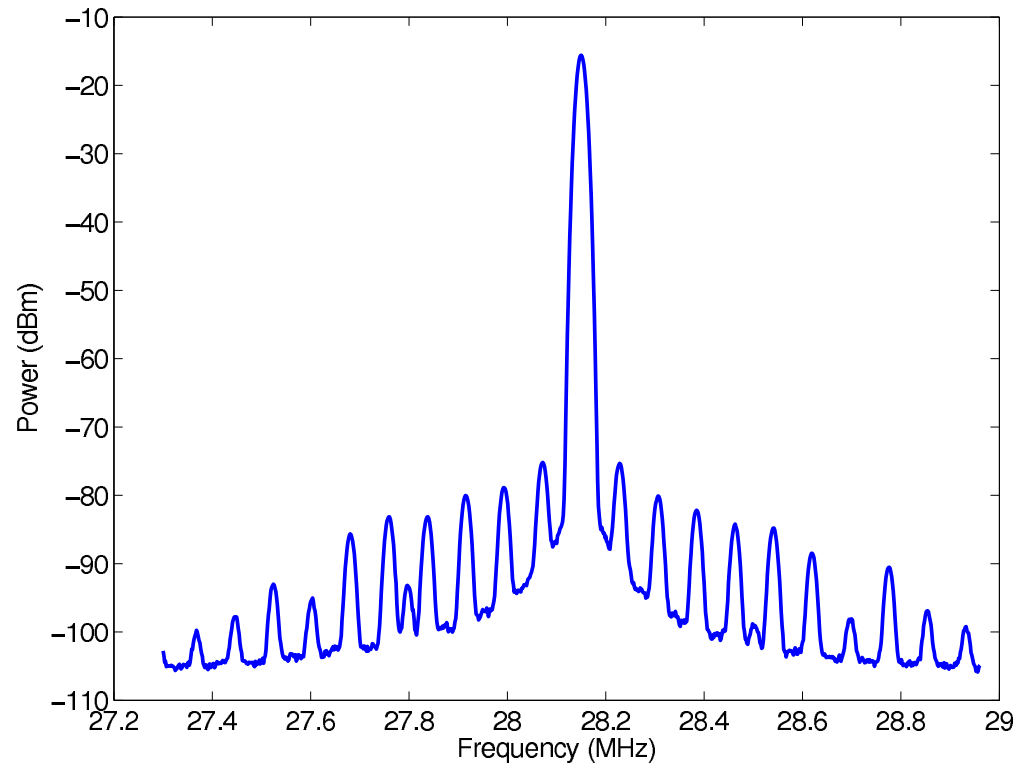

Figure 3: Blue ring spectrum near the fundamental mode of the $28 \mathrm{MHz}$ cavity. 

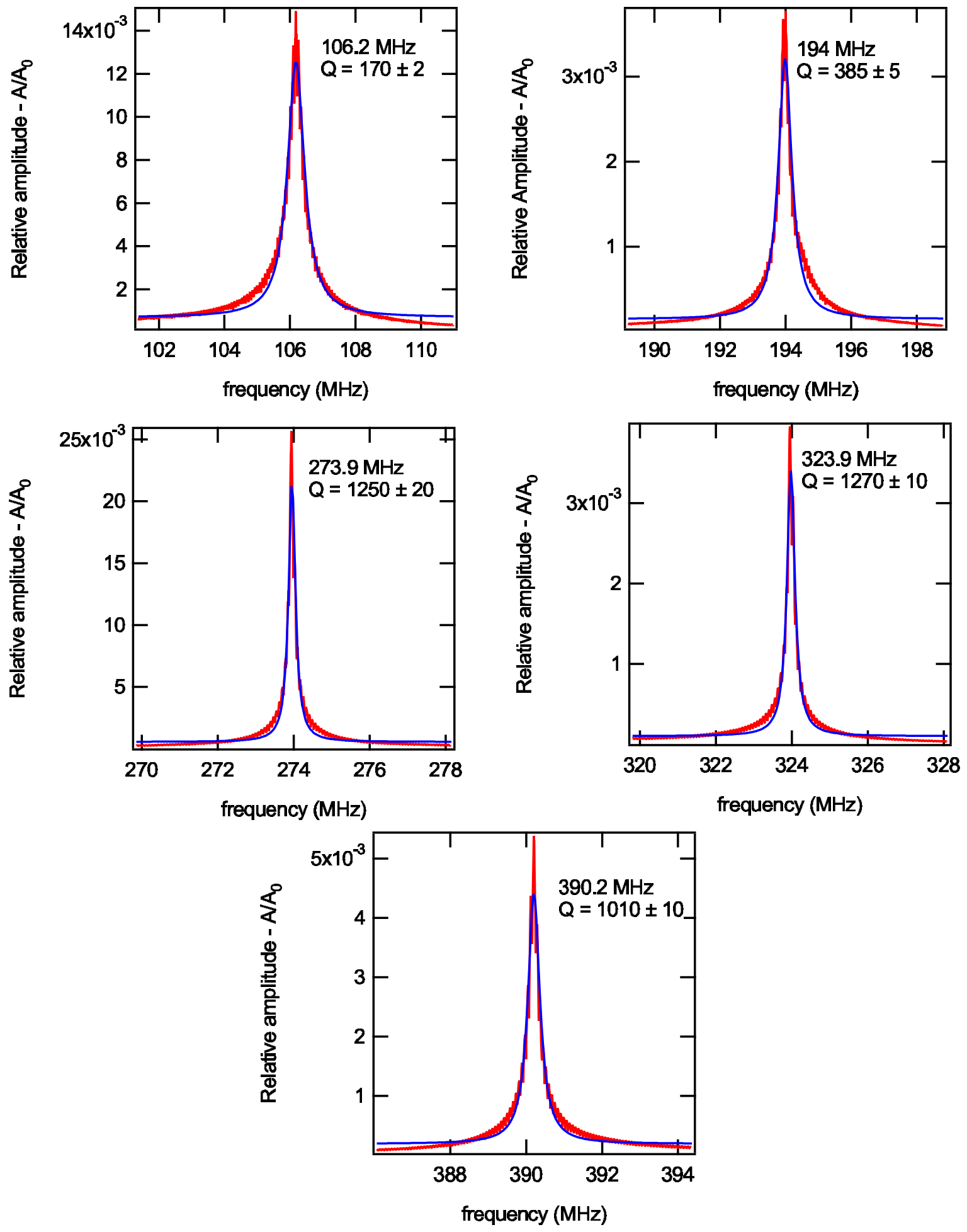

Figure 4: Measured loaded $\mathrm{Q}$ factor and center frequencies for the HOMs in the $28 \mathrm{MHz}$ cavity. The blues line is the measured spectrum and the red line is the fitting. 
Table 3: Summary of measured HOM frequencies for the $28 \mathrm{MHz}$ and comparison with a previous measurement (26.1 MHz cavity) [2]

\begin{tabular}{cccc}
\hline \hline \multicolumn{2}{c}{$28 \mathrm{MHz}$} & \multicolumn{2}{c}{$26.7 \mathrm{MHz}$} \\
$\mathrm{f}(\mathrm{MHz})$ & $\mathrm{Q}_{L}$ & $\mathrm{f}(\mathrm{MHz})$ & $\mathrm{Q}_{L}$ \\
\hline 106.2 & $170 \pm 2$ & 98.8 & 190 \\
193.98 & $385 \pm 5$ & 216.3 & 460 \\
273.9 & $1250 \pm 20$ & 287.4 & 1500 \\
323.9 & $1270 \pm 10$ & 342.2 & 2100 \\
390.2 & $1010 \pm 10$ & 402.2 & 1100 \\
\hline \hline
\end{tabular}

the spectrum lines for the yellow case are always bellow the blue lines in Fig. 2

In order to get a cleaner spectrum we measured the HOM damper spectrum at injection energy with a single bunch in the machine with an intensity of $N_{b}=1.5 \times 10^{11}$ protons. In order to measure the center frequencies and $\mathrm{Q}$ factors of the HOMs, we fitted this spectrum to the following Lorentzian formula

$$
A(\omega)=A_{0}\left[1+Q_{L}^{2}\left(\frac{\omega}{\omega_{0}}-\frac{\omega_{0}}{\omega}\right)^{2}\right]^{-1}
$$

where $Q_{L}$ is the mode loaded quality factor and $\omega_{0}$ the center frequency. The summary of the measured HOM frequencies and $\mathrm{Q}$ values is shown in Table 3 and are compared with a previously measured data. The HOM frequencies are identified to be very close to what had been measured and the Q vales are close to those reported on [2]. The measured and fitted data for each mode are in Fig. 4.

\section{Analytic calculation of HOM power}

In order to calculate the power in each $\mathrm{HOM}$ of the $28 \mathrm{MHz}$ cavity first we have to take into account the frequency spectrum of the bunches at the moment of the measurement. For the proton run in 2008 the filling pattern used was: 109 bunches with a gap between buckets 38 and 41 and an abort gap from buckets 112 to 120. Also we have to consider that the intensity and bunch shape varies along the bunch train, as shown in Fig. 5 and 6 . For simplicity we will consider that all the bunches have an average current of $1.6 \times 10^{11}$ protons, with a rms bunch length of $5 \mathrm{~ns}$ with the exception of the Artus bunch that has one third of the intensity of the other bunches. Furthermore we will consider that all bunches can be approximated by a Gaussian shape, which turns out to be a good approximation as shown in Fig. 6.

In the time domain the beam current is given by:

$$
I(t)=\sum_{k=-\infty}^{\infty} I_{0}\left(t-k T_{0}\right)=\sum_{k=-\infty}^{\infty} \sum_{h=1}^{N_{1}} \frac{q_{h}}{\sqrt{2 \pi \sigma_{t}^{2}}} \exp \left[-\frac{\left(t-k T_{0}-h T_{0} / N_{1}\right)^{2}}{2 \sigma_{t}^{2}}\right]
$$

where $q_{h}$ is the charge per bunch, $T_{0}$ is the revolution period and $N_{1}$ is the number of buckets which are 120 total in RHIC. The periodic current function can be expanded into 


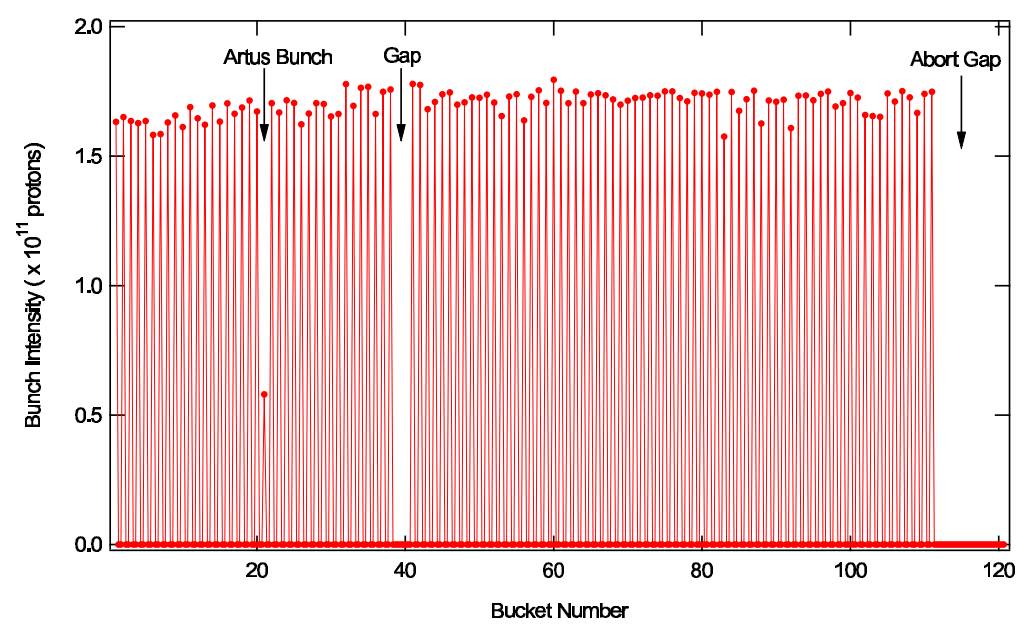

Figure 5: Filling pattern in the Blue ring during the measurement of the HOM power distribution.
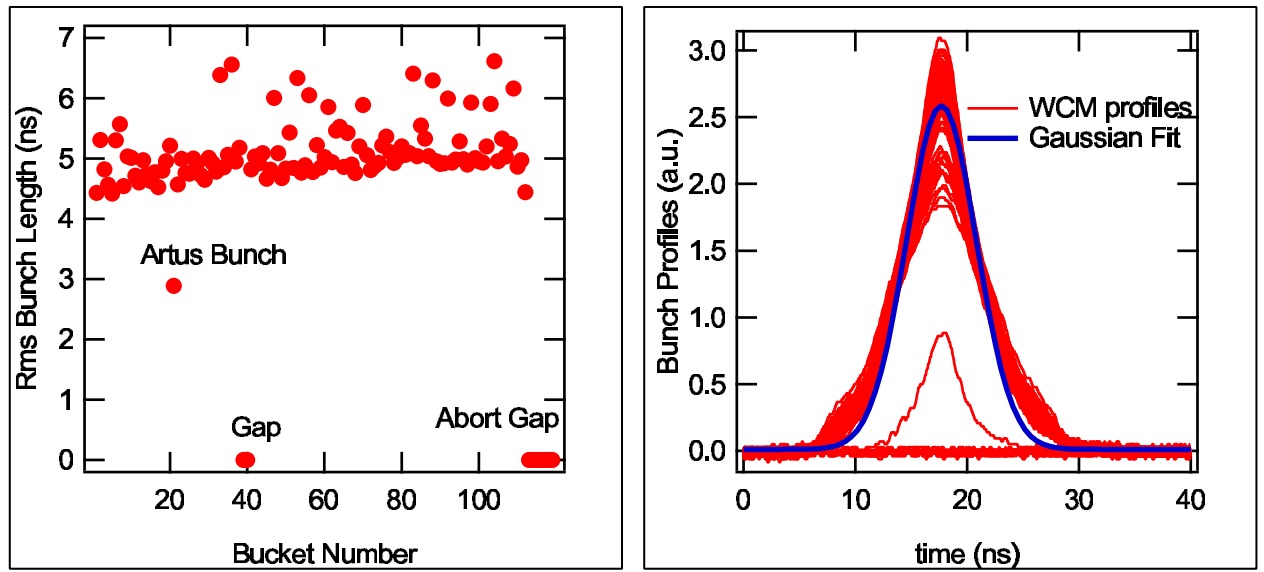

Figure 6: Filling pattern in the Blue ring during the measurement of the HOM power distribution. 
Table 4: Summary of the measured $28 \mathrm{MHz}$ cavity HOM frequencies with simulated $R / Q[2]$ and the calculated beam frequencies at store. The dissipated power of each HOM is also calculated with two different assumptions: $\mathrm{P}$ (point charge) assumes trains of point charge bunches and $\mathrm{P}$ (Gaussian) assumes a train of Gaussian bunches. We considered 109 bunches with $1.2 \times 10^{11}$ protons/bunch.

\begin{tabular}{c|c|c|c|c}
\hline \hline $\begin{array}{c}\mathrm{f} \\
(\mathrm{MHz})\end{array}$ & $\begin{array}{c}\mathrm{f}_{\text {beam }} \\
(\mathrm{MHz})\end{array}$ & $\begin{array}{c}R / Q \\
(\Omega)\end{array}$ & $\begin{array}{c}\mathrm{P}(\text { Gaussian }) \\
(\mathrm{mW})\end{array}$ & $\begin{array}{c}\mathrm{P} \text { (Measured) } \\
(\mathrm{mW})\end{array}$ \\
\hline 28.149 & 28.149 & 67.6 & 3.35 & 4 \\
106.193 & 103.215 & 2.8 & 0.36 & 0.4 \\
193.923 & 197.046 & 0.12 & 0.0015 & 0.012 \\
273.925 & 272.111 & 3.6 & 0.0062 & 0.004 \\
323.93 & 319.027 & 9.4 & 0.0115 & $4 \times 10^{-4}$ \\
390.187 & 384.709 & 2.8 & 0.003 & $2.5 \times 10^{-5}$ \\
\hline \multicolumn{3}{c|}{ Total Power } & 3.7 & 4.4 \\
\hline \hline
\end{tabular}

a Fourier Series with the following spectrum components

$$
I_{n}=\frac{1}{T_{0}} \int_{0}^{T_{0}} I_{0}(t) \exp \left(j \frac{2 \pi n}{T_{0}} t\right) \approx \sum_{h=1}^{N_{1}} \frac{q_{h}}{T_{0}} \exp \left(j \frac{2 \pi n h}{T_{0}}\right) \exp \left[-\left(\frac{2 \pi n}{T_{0}}\right)^{2} \frac{\sigma_{t}^{2}}{2}\right]
$$

The real part of the impedance of the $\mathrm{k}^{\text {th }} \mathrm{HOM}$ is characterized by the resonant frequency $\omega_{k}$, the quality factor $Q_{k}$ and the shunt impedance $R_{k}$ and is given by

$$
\operatorname{Real}[Z(\omega)]=\frac{(R / Q)_{k} Q_{k}}{1+Q_{k}^{2}\left(\frac{\omega}{\omega_{r}}-\frac{\omega_{r}}{\omega}\right)^{2}}
$$

Then the beam power delivered by the $\mathrm{n}^{\text {th }}$ beam harmonic in the $\mathrm{k}^{\text {th }}$ cavity mode is

$$
P_{n, k}=\frac{2(R / Q)_{k} Q_{L k} I_{n}^{2}}{1+Q_{L k}^{2}\left(\frac{\omega}{\omega_{r}}-\frac{\omega_{r}}{\omega}\right)^{2}}
$$

where $Q_{L k}$ is the loaded quality factor of the considered mode. The total power released by the beam due to a specific mode is the sum of the power released by each beam line

$$
P_{k}=\sum_{n=0}^{\infty} \frac{2(R / Q)_{k} Q_{L k} I_{n}^{2}}{1+Q_{L k}^{2}\left(\frac{\omega}{\omega_{r}}-\frac{\omega_{r}}{\omega}\right)^{2}}
$$

Table 4 summarizes the HOM frequencies, their respective $R / Q$ value which comes from a MAFIA simulation [2], the beam bunch frequency harmonics that are closer to the HOM frequencies and the calculated power dissipated in the HOM damper. The beam frequencies are calculated using the bunch frequency, which is $9.383 \mathrm{MHz}$, multiplied by integers and we considered a full machine (109 bunches) with and average intensity of $1.6 \times 10^{11}$ protons/bunch (Fig. 5). The measured frequencies show that there is no significant overlap between the beam frequencies and the cavity mode frequencies. The individual power in each HOM is calculated using Eq. 6 and the $28 \mathrm{MHz}$ mode is considered 
to be rejected by the HOM filter by $-60 \mathrm{~dB}$ [1]. The summed-up HOM power is around $3.7 \mathrm{~mW}$ for the Gaussian bunch and the sum of the measured power in each HOM is 4.4 $\mathrm{mW}$.

Comparing the measured HOM power of $6.5 \mathrm{~mW}$ with the calculated HOM power of $3.7 \mathrm{~mW}$ we see that the calculated HOM power in the HOM are about half of the total measured power, this difference could be explained by the power distributed in many bunches lines around each HOM line, as show in Fig. 3 which is around $2 \mathrm{~mW}$ if we consider the total power measured for each HOM (Table $4-6.5-4.4=2.1 \mathrm{~mW}$ ). The other $0.7 \mathrm{~mW}$ is due to a discrepancy between the calculated and the measured power for the fundamental mode. This is most likely due to the power rejection at the fundamental mode that was used based on the design value of the HOM damper [2]. In this case we considered that the fundamental had a $60 \mathrm{~dB}$ rejection from the HOM filter and a small difference (about $1 \mathrm{~dB}$ ) in this value could account for the difference in the power calculated and measured.

\section{Summary}

We have measured the HOM power of the RHIC $28 \mathrm{MHz}$ RF system and identified each HOM frequencies and respective $Q_{L}$ factors. The total HOM power delivered to the HOM damper was measured to be around $6.5 \mathrm{~mW}$. The values of the frequencies and $\mathrm{Q}$ factors for the $28 \mathrm{MHz}$ and $197 \mathrm{MHz}$ systems are comparable to the previously measured and simulated numbers reported in $[1,2]$ and show a very good agreement. The calculated HOM power is around $3.7 \mathrm{~mW}$, which is smaller than the measured power of $6.5 \mathrm{~mW}$. The discrepancy between the measurement and the calculation may be attributed to a slightly lower number in the rejection filter of the fundamental frequency than the original design value and the power dissipated in the bunch lines which is not taken into account in the calculation.

\section{Acknowledgement}

The authors would like to thank Mike Brennan and Mike Blaskiewicz for their advice and helpful discussion.

\section{References}

[1] James Rose, "Calculations of HOMs and Coupled Bunch Instabilities due to the RHIC rf Cavities", EPAC proceedings, London, England, June 27-July 1, 1994.

[2] S. Kwiatkowski et al., "HOM damper for RHIC $26.7 \mathrm{MHz}$ accelerating system",RHIC/RF Technical Note No. 25, Jan. 1995.

[3] Padamsee book, p 338. 